\title{
Fast-ion transport in low density L-mode plasmas at TCV using FIDA spectroscopy and
} the TRANSP code

Geiger, B.; Karpushov, A.; Duval, B. P.; Marini, C.; Sauter, O.; Andrebe, Y.; Testa, D.; Schneider, P.A.; Salewski, Mirko

Published in:

Plasma Physics and Controlled Fusion

Link to article, DOI:

10.1088/1361-6587/aa8340

Publication date:

2017

Document Version

Peer reviewed version

Link back to DTU Orbit

Citation (APA):

Geiger, B., Karpushov, A., Duval, B. P., Marini, C., Sauter, O., Andrebe, Y., Testa, D., Schneider, P. A., \& Salewski, M. (2017). Fast-ion transport in low density L-mode plasmas at TCV using FIDA spectroscopy and the TRANSP code. Plasma Physics and Controlled Fusion, 59(11), [115002]. https://doi.org/10.1088/1361$6587 /$ aa8340

\section{General rights}

Copyright and moral rights for the publications made accessible in the public portal are retained by the authors and/or other copyright owners and it is a condition of accessing publications that users recognise and abide by the legal requirements associated with these rights.

- Users may download and print one copy of any publication from the public portal for the purpose of private study or research.

- You may not further distribute the material or use it for any profit-making activity or commercial gain

- You may freely distribute the URL identifying the publication in the public portal 


\section{PAPER}

\section{Fast-ion transport in low density L-mode plasmas at TCV using FIDA spectroscopy and the TRANSP code}

To cite this article: B Geiger et al 2017 Plasma Phys. Control. Fusion 59115002

\section{Manuscript version: Accepted Manuscript}

Accepted Manuscript is "the version of the article accepted for publication including all changes made as a result of the peer review process, and which may also include the addition to the article by IOP Publishing of a header, an article ID, a cover sheet and/or an 'Accepted

Manuscript' watermark, but excluding any other editing, typesetting or other changes made by IOP Publishing and/or its licensors"

This Accepted Manuscript is ( @ @ 2017 Max-Planck-Institut fuer Plasmaphysik.

During the embargo period (the 12 month period from the publication of the Version of Record of this article), the Accepted Manuscript is fully protected by copyright and cannot be reused or reposted elsewhere.

As the Version of Record of this article is going to be / has been published on a subscription basis, this Accepted Manuscript is available for reuse under a CC BY-NC-ND 3.0 licence after the 12 month embargo period.

After the embargo period, everyone is permitted to use copy and redistribute this article for non-commercial purposes only, provided that they adhere to all the terms of the licence https://creativecommons.org/licences/by-nc-nd/3.0

Although reasonable endeavours have been taken to obtain all necessary permissions from third parties to include their copyrighted content within this article, their full citation and copyright line may not be present in this Accepted Manuscript version. Before using any content from this article, please refer to the Version of Record on IOPscience once published for full citation and copyright details, as permissions will likely be required. All third party content is fully copyright protected, unless specifically stated otherwise in the figure caption in the Version of Record.

View the article online for updates and enhancements. 


\title{
Fast-ion transport in low density L-mode plasmas at TCV using FIDA spectroscopy and the TRANSP code
}

B. Geiger ${ }^{1}$, A. N. Karpushov², B.P. Duval ${ }^{2}$, C. Marini² ${ }^{2}$ O. Sauter ${ }^{2}$, Y. Andrebe ${ }^{2}$, D. Testa $^{2}$, M. Marascheck ${ }^{1}$, M. Salewski ${ }^{3}$, P.A. Schneider ${ }^{1}$, the TCV Team ${ }^{4}$ and the EUROfusion MST1 Team ${ }^{5}$

${ }^{1}$ Max-Planck-Institute for Plasma Physics, Boltzmannstr. 2, 85748 Garching, Germany

${ }^{2}$ Swiss Plasma Center, EPFL, Lausanne, Switzerland

${ }^{3}$ Technical University of Denmark, Dk-2800 Kgs. Lyngby, Denmark

${ }^{4}$ See author list of S. Coda et al 2017 Nucl. Fusion 57102011

${ }^{5}$ See author list of H. Meyer et al 2017 Nucl. Fusion 57102014

Corresponding Author: benedikt.geiger@ipp.mpg.de

\begin{abstract}
:
Experiments with the new neutral beam injection (NBI) source of TCV have been performed with high fast-ion fractions $(>20 \%)$ that exhibit a clear reduction of the loop voltage and a clear increase of the plasma pressure in on- and off-axis heating configurations. However, good quantitative agreement between the experimental data and TRANSP predictions is only found when including strong additional fast-ion losses. These losses could in part be caused by turbulence or MHD activity as, e.g. high frequency modes near the frequency of toroidicity induced Alfvén Eignmodes (TAEs) are observed. In addition, a newly installed Fast-Ion D-Alpha (FIDA) spectroscopy system measures strong passive radiation and, hence, indicates the presence of high background neutral densities such that charge-exchange losses are substantial. Also the active radiation measured with the FIDA diagnostic, as well as data from a neutral particle analyzer, suggest strong fast-ion losses and large neutral densities. The large neutral densities can be justified since high electron temperatures (3-4 keV), combined with low electron densities (about $2 \times 10^{19} / \mathrm{m}^{3}$ ) yield long mean free paths of the neutrals which are penetrating from the walls.
\end{abstract}

\section{Introduction}

The behavior of fast, suprathermal particles in high temperature plasmas must be understood in view of future fusion reactors. Fast ions result from the fusion reaction and can be generated additionally through neutral beam injection (NBI) or ion cyclotron resonance heating $(\mathrm{ICRH})$. The fast particles heat the background plasma via collisions with ions and electrons and can, in case of an anisotropic fast-ion velocity space distribution, drive 
non-inductive toroidal currents. In the absence of plasma instabilities (and other anomalous transport processes), the fast-ion slowing down distribution is neoclassical and can be modeled by solving the Fokker-Planck equation, e.g. with the TRANSP/NUBEAM code package [1, 2]. However, high temperature plasmas are not instability-free and phenomena such as Alfvén eigenmodes [3], sawtooth crashes [4, 5] or small-scale turbulence [6] cause, so-called, anomalous transport. During off-axis neutral beam experiments at MAST [7] and ASDEX Upgrade [8] for example, the current drive efficiency is not as expected by neoclassical theory and an anomalous fast-ion diffusion was needed to explain the data. Furthermore, strong fast-ion losses are observed e.g. at ASDEX Upgrade [9] or DIII-D [10] when Alfvén Eigenmodes are present. The modes are even supposed to exhibit a critical fast-ion density gradient behavior that was recently observed at DIII-D [11].

The impact of the anomalous transport on the fast-ion distribution function must be considered when designing and studying the concept of future devices. It is, thus, essential to verify the theoretical models that describe the fast-ion transport based on reliable experimental test cases. Here, fast-ion experiments at the TCV tokamak are particularly interesting as this device operates in a unique region of parameter space. The small size of TCV, combined with powerful NBI heating systems, result in high fast-ion densities which can drive e.g. Alfvén Eigenmodes unstable. Moreover, the combination of relatively low energetic fast particles $(\mathrm{E}=25 \mathrm{keV}$ injection energy) and high electron temperatures $\left(\mathrm{T}_{\mathrm{e}}\right)$, achievable by additional ECRH heating, is an ideal case to study the turbulent fast-ion transport. The latter is expected to become stronger with deceasing values of $\mathrm{E} /\left(\mathrm{T}_{\mathrm{e}}\right)$ [12]. Fast-ion experiments at TCV can, therefore, be an ideal test case for different codes such as Hagis [13], Gene [14] or ASCOT [15] that allow modeling the various aspects of anomalous fast-ion transport.

This paper is structured as follows. Section 2 provides details on the experimental setup at TCV, including a newly installed FIDA system. In section 3 the implementation of the TRANSP code and FIDASIM at TCV are described. Section 4 describes the experimental results using $1 \mathrm{MW}$ of on-axis and off-axis NBI. Comparison between measured loop voltages and toroidal beta values with TRANSP modeling are given and experimental FIDA spectra are compared with synthetic spectra from FIDASIM. In addition, the magnetic fluctuations observed during the experiments and most likely caused by Alfvén Eigenmodes are analyzed. Finally, a short summary and discussion are provided in section 5 .

\section{Experimental Setup}

The Tokamak à Configuration Variable (TCV) is a mid-size machine with major and minor radii of $0.88 \mathrm{~m}$ and $0.25 \mathrm{~m}$, respectively. Magnetic field strengths of up to $1.5 \mathrm{~T}$ and currents of up to $1 \mathrm{MA}$ are applied and carbon is the dominant wall material. During 

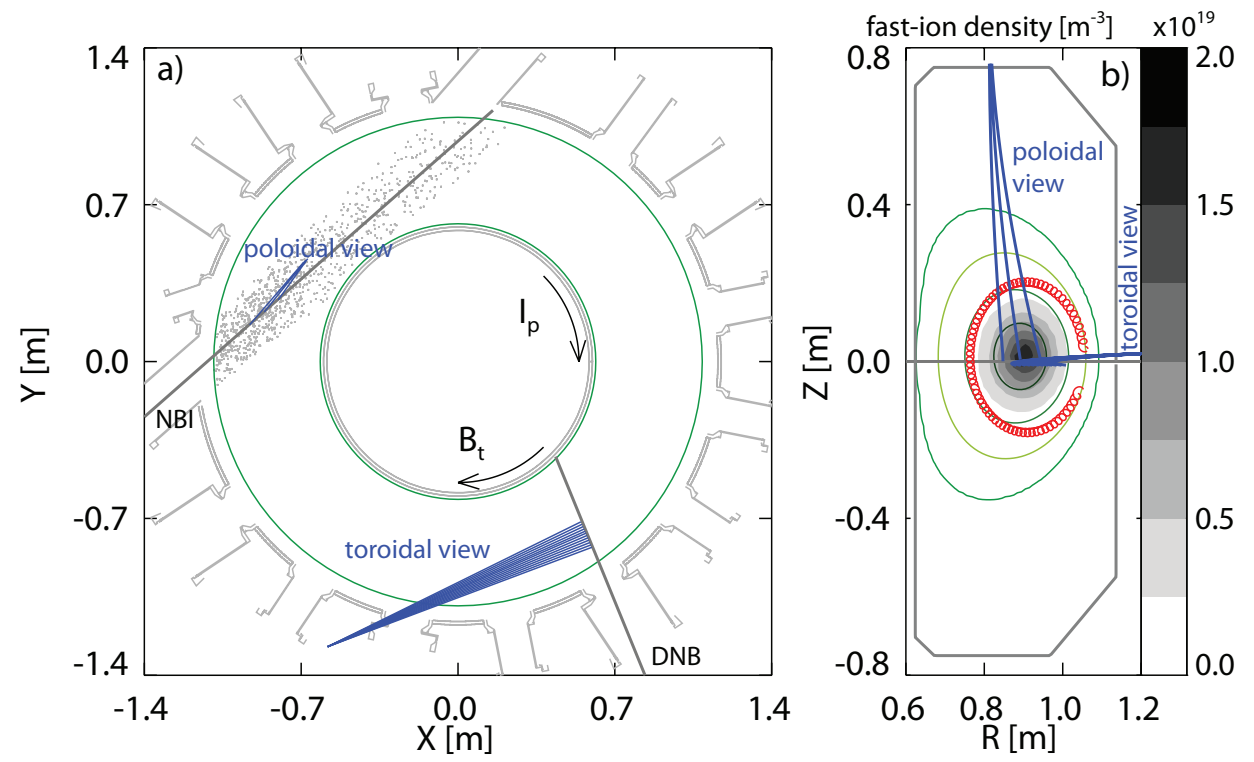

FIG. 1: Horizontal (a) and vertical (b) cuts through the TCV tokamak. The birth positions of NBI markers from a TRANSP simulation for the heating beam are illustrated in (a) by gray points. In (b), a fast-ion orbit is plotted in red that corresponds to a fast ion with $25 \mathrm{keV}$ and a pitch value of 0.9 at the low field side in discharge \#53783. The line of sight geometry of the FIDA diagnostic is plotted in blue in both figures.

the experiments discussed herein, up to 1.5 MW of electron cyclotron resonance heating (ECRH) were available from three launchers, operated in second harmonic X-mode at 82.7 GHz [16]. In addition, a neutral heating beam with $1 \mathrm{MW}$ of nominal power was available, injecting deuterium atoms tangentially through the plasma with a full energy of $25 \mathrm{keV}$ [17]. The geometry of the NBI source and an orbit of a corresponding $25 \mathrm{keV}$ ion with a pitch of $p=v_{\|} / v=0.9$ (at the low field side) are plotted in figure 1 .

Ion temperature and rotation measurements at TCV employ charge exchange recombination spectroscopy (CXRS) on carbon ions, using a separate diagnostic neutral beam (DNB) [18. The DNB has a power of about $60 \mathrm{~kW}$ and injects hydrogen neutrals at $\sim 49 \mathrm{keV}$. It is typically operated with $12 \mathrm{~ms}$ duration beam blips, separated by $24 \mathrm{~ms}$ to obtain accurate background subtraction for the CXRS measurement. The electron density and temperature were measured by a Thomson scattering system [19] that has 47 vertically distributed lines of sight, focused on a laser which transits from the bottom to the top of the machine. The system is typically operated with a temporal resolution of $\sim 17 \mathrm{~ms}$ and can provide full profiles for a wide range of vertical plasma displacements.

For fast particle studies, TCV is equipped with a compact neutral particle analyzer (CNPA) [20] that measures the flux of neutrals with energies up to $30 \mathrm{keV}$ that originate from charge exchange reactions between fast ions and neutrals in the plasma. 

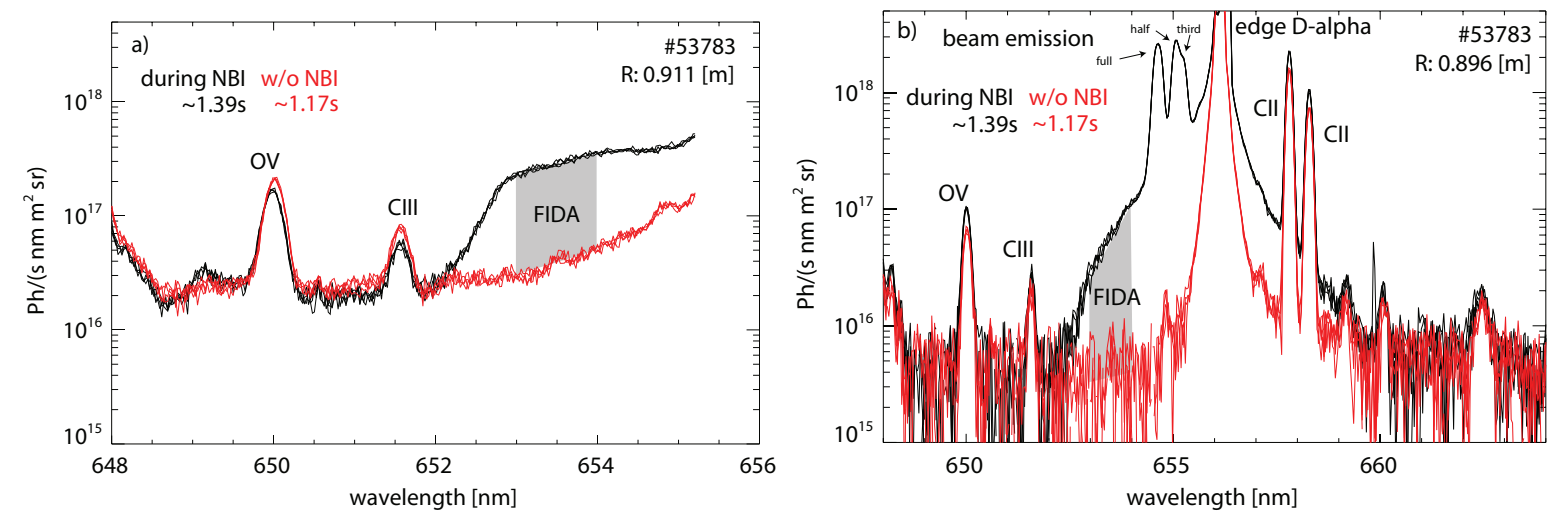

FIG. 2: Measured spectra from a central toroidal (a) and from a central poloidal (b) line of sight without (red) and with (black) the heating beam. Four subsequently measured spectra are plotted per time-point that have been acquired with 3 ms exposure time.

\subsection{FIDA spectroscopy}

In addition, a newly installed FIDA system is available, which measures Doppler shifted Balmer alpha radiation, emitted by the neutralized fast ions (based on the principle developed by [21]). For the experiments discussed here, two observation geometries are employed that are shown in blue in figure 1. Firstly, an array of toroidal lines of sight (toroidal view) is used that originally belonged to the CXRS system [22] and intersects the diagnostic beam (DNB). Secondly, a new system was installed that observes the heating beam from above (close to poloidal view). Each view is connected to a Czerny turner like spectrometer that uses two lenses with a focal length of $200 \mathrm{~mm}$, a reflection grating and an EM-CCD camera (ANDOR, iXon, $512 \times 512$ pixels), operated with a time resolution of $3 \mathrm{~ms}$.

Measured spectra for one representative channel of each FIDA system are shown in figure 2. As can be seen, the toroidal system is set up to observe only wavelengths up to $655 \mathrm{~nm}$. This avoids the unshifted and highly intense passive D-alpha line at $656.1 \mathrm{~nm}$ that would cause strong detector saturation. The poloidal view, instead, observes the complete D-alpha spectrum with only modest saturation at $656.1 \mathrm{~nm}$ since less passive light is collected. In contrast to the toroidal view, the poloidal lines of sight feature a shorter path through the plasma $(\sim 80 \mathrm{~cm}$ instead of $\sim 130 \mathrm{~cm})$ and are less tangential to the edge region where the density of cold neutrals, emitting passive radiation, is largest.

The spectra in red in figure 2 are without NBI while those in black are during NBI. A broad spectral component of Doppler shifted D-alpha light (FIDA) is present in both spectra with NBI. In addition, the poloidal measurement exhibits three peaks from the beam emission (full, half and one third energy component) near $655 \mathrm{~nm}$. In the observed wavelength range of the toroidal system, the beam emission cannot be resolved as the Doppler shift is too small (the lines of sight are almost perpendicular to the DNB). Even 

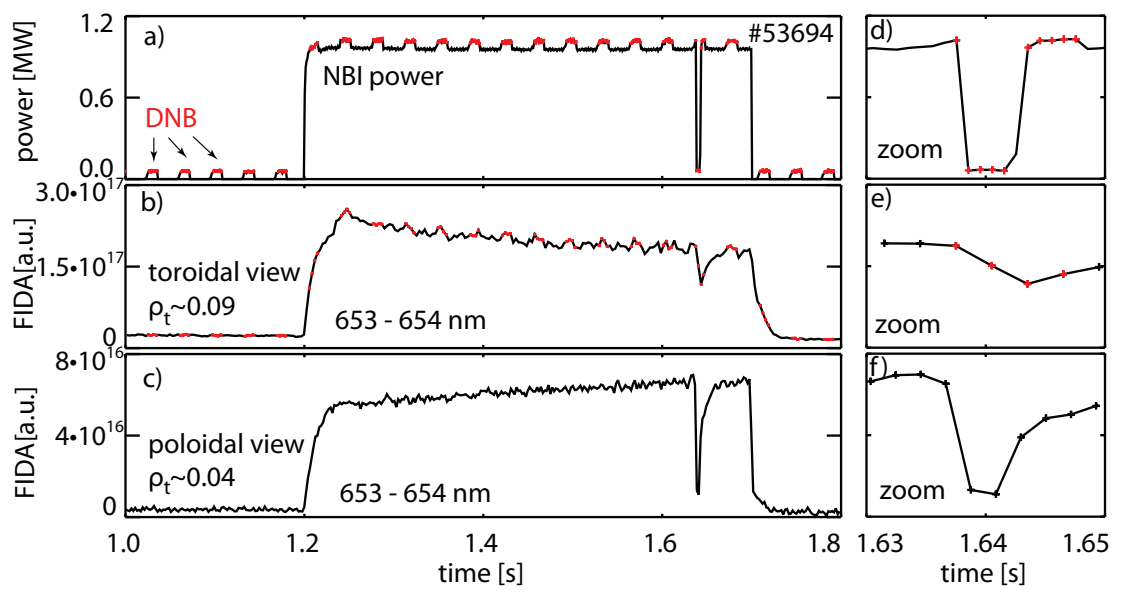

FIG. 3: Temporal evolution of the integrated FIDA signal (the integration range is illustrated in gray in figure 2), measured with the toroidal and poloidal systems. Phases when the diagnostic beam is on are indicated in red. A zoom of figures $a, b$ and $c$ during the beam power notch is illustrated in figures $d$, $e$ and $f$

if the Doppler shift was larger, no beam emission and also no active FIDA radiation are expected in the presented spectrum since it was acquired with the DNB off. The presence of a broad spectral feature can, hence, only be explained by passive FIDA radiation, emitted by fast ions after charge exchange reactions on background neutrals. The intensity of this passive signal is stronger than the active FIDA component that originates from charge exchange reactions with neutrals present along the DNB path. To illustrate this, figure 3 shows the evolution of the integrated FIDA radiation in discharge \#53694 between $653 \mathrm{~nm}$ and $654 \mathrm{~nm}$ from which an offset has been subtracted (determined at $\sim 651 \mathrm{~nm}$ ). The FIDA intensity, measured by the toroidal view (figure $3 \mathrm{~b}$ ), increases when the NBI is turned on and becomes modulated slightly with the DNB power $(\sim 5 \%)$. At about $1.63 \mathrm{~s}$, the heating beam is turned off for $6 \mathrm{~ms}$ (see figure $3 \mathrm{a}$ and $\mathrm{d}$ ). This causes a modest drop in the toroidal signal, explained by its passive nature and by the comparably long confinement time $(\approx 10 \mathrm{~ms}$ ) of fast ions (The calculated slowing down time for fast ions with $25 \mathrm{keV}$ is $\sim 60 \mathrm{~ms}$. However, as discussed later, charge exchange losses are significant at TCV which reduce the fast-ion confinement time to about $10 \mathrm{~ms}$ when considering a realistic density of background neutrals.). In contrast, the poloidal measurement (figure 3r) is strongly affected by the beam power notch. Despite the presence of fast ions due to the long confinement time, no active radiation is available along the poloidal line of sight when the heating beam is off. The strong drop of the poloidal signal is due to the dropping active component and, thus, proves that this measurement is dominated by active radiation. Compared with the toroidla view, the direct interpretation of poloidal spectra is therefore easier.

The two viewing geometries allow investigating different parts of the fast-ion velocity 

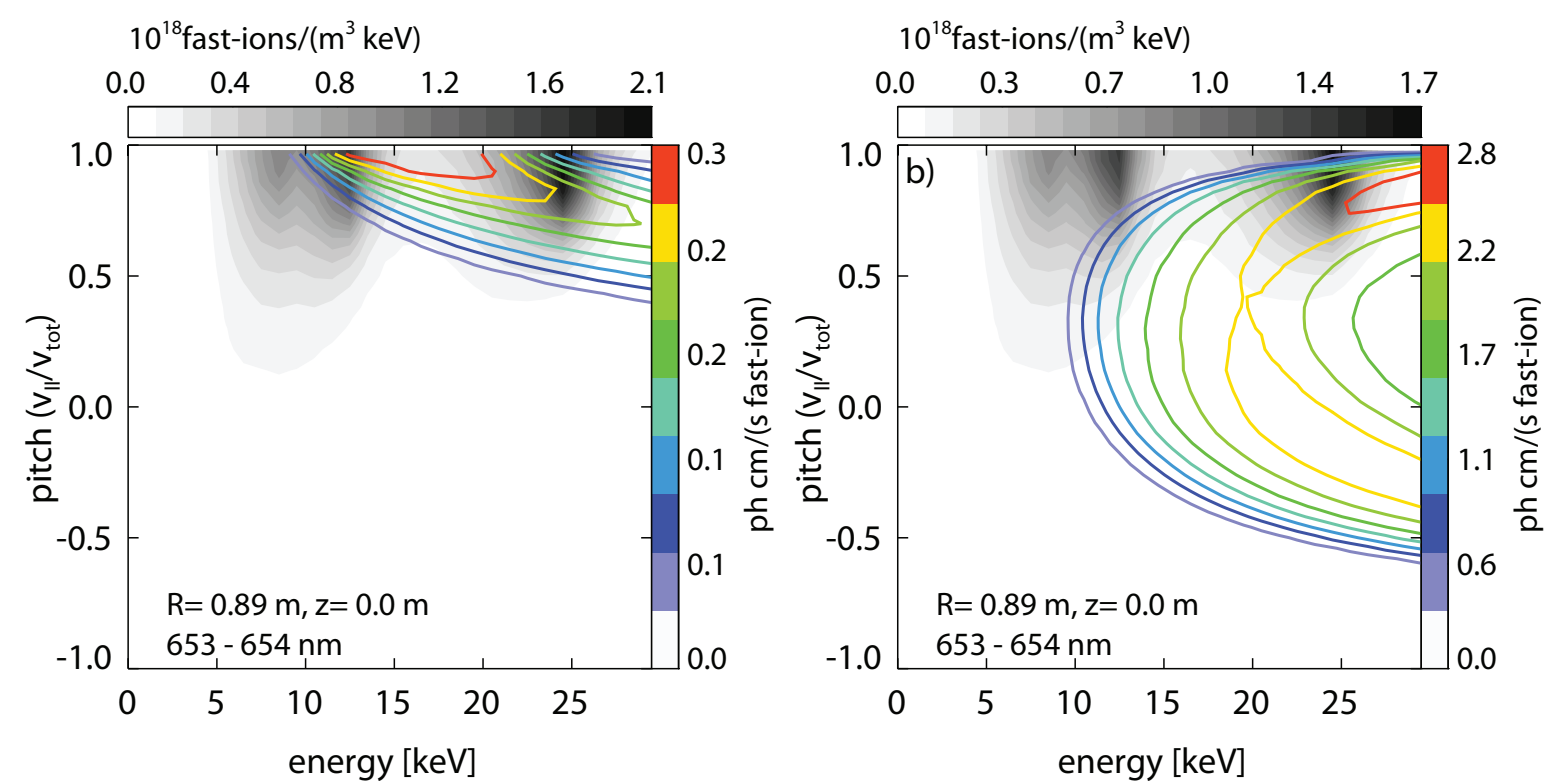

FIG. 4: Weight functions showing the observed part of the fast-ion velocity space distribution when analyzing the wavelength region between $653 \mathrm{~nm}$ and $654 \mathrm{~nm}$ (see figure Q) using the toroidal view (a) and the poloidal view (b). In addition, the fast-ion velocity distribution of discharge \#53783 at $1.39 \mathrm{~s}$ is plotted which is present along the corresponding line of sight.

space as illustrated by the weighting functions [23] in figure 4. Here weight functions are displayed for the wavelength range between $653 \mathrm{~nm}$ and $654 \mathrm{~nm}$, which corresponds to a spectral region where FIDA radiation is well accessible. As can be seen, the toroidal view is sensitive to co-rotating fast ions at this blue-shifted wavelength range, while the poloidal view is more sensitive to fast ions with small pitch values. Thus, fast-ion transport studies in different parts of the velocity space can be performed.

\section{Modeling}

\subsection{TRANSP}

The TRANSP code [1] has been applied in its interpretative mode to compute neoclassical fast-ion distribution functions and to calculate quantities such as the loop voltage or the toroidal beta. TRANSP uses the NUBEAM package to model the heating and current drive from NBI and uses the TORAY 24] code for ECRH and ECCD. It requires the evolution of the kinetic profiles, plasma current, toroidal magnetic field, the shape of the last closed flux surface and an initial q-profile. TRANSP then applies a time dependent equilibrium solver that considers the plasma pressure, neutral beam current drive, electron cyclotron current drive and the bootstrap current. Herein, the NBI current drive 

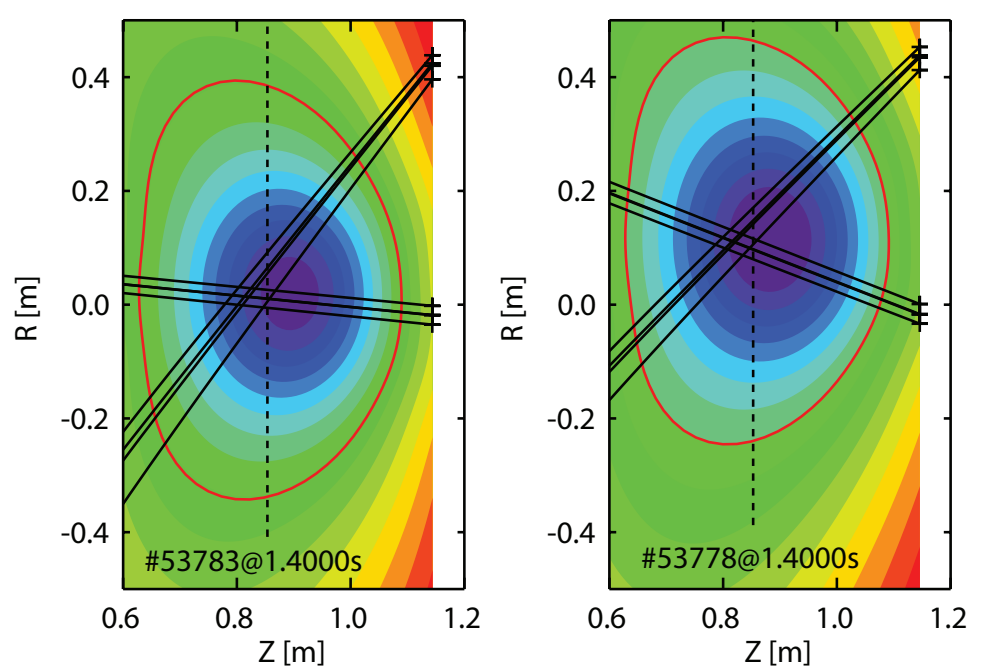

FIG. 5: Poloidal flux surfaces as calculated by TRANSP with increasing values from the center to the plasma edge. The ECRH ray geometry for discharges \#53783 (on-axis) and \#53r78 (off-axis) is shown in black. The dashed vertical lines illustrate the resonance position of the ECRH radiation (82.7 GHz).

efficiency is calculated according to reference [25] with the bootstrap current and the plasma resistivity determined according to reference [26].

For TORAY, each ECRH launcher at TCV is modeled from four injection lines, each simulated by 100 rays to account for the divergence. For the experiments discussed here, a divergence of eight degrees is employed that is significantly larger than the actual divergence but is used to account for the broadening of the ECRH deposition profile by radial transport of suprathermal electrons as reported by [27]. If this effect was not considered, the equilibrium solver of TRANSP fails due to too strong and localized currents. The geometry of the injection lines of two launchers are plotted in figure 5 for two vertical plasma positions (on-axis NBI and off-axis NBI, see below).

NUBEAM calculates the NBI deposition profile, i.e. the initial location of fast ions, and subsequently solves the Fokker-Planck equation with a Monte Carlo approach in two spatial and two velocity space coordinates (R, Z, energy, pitch). It employs the NBI power, energy, species mix $(68 \%, 25 \%, 7 \%$ for the full, half and one third energy, here given in terms of the power) and the geometry, focus and divergence of all NBI sources. The beam species must be specified, i.e. deuterium for the heating beam and hydrogen for the diagnostic beam. We assume a rectangular shape of the heating beam even though the source is circular. This is necessary as the beam divergence is different in the horizontal and vertical planes (1.13 degree and 0.81 degree, respectively) which cannot, as yet, be simulated by NUBEAM. NUBEAM can either model a circular source with one divergence or a rectangular source with separate divergences. It should be noted that the impact of this modification has been checked and is very small. The assumption of a circular source 
with an average divergence yields very similar results.

Figure 1 1 illustrates resulting deposition positions of NBI markers for a simulation that is based on discharge \#53783 (discussed in detail in section 4) with on-axis NBI. The predicted poloidal fast-ion distribution is plotted in figure $1 \mathrm{~b}$ and exhibits a clear peak in the center. A corresponding velocity space distribution is shown in figure 4 with most fast ions at pitch values close to one. The injection peaks at full, half and one third energy can be identified.

Fast-ion orbit losses are considered in TRANSP by approximating the first wall using 8 lines in the poloidal plane as shown in figure 1b in gray. All fast ion orbits that would intersect these lines are treated as lost.

To consider charge exchange losses with background neutrals in TRANSP, the background neutral density is modelled by FRANTIC [28], a 1D code that describes the penetration of neutrals sourced at the walls into the plasma. In addition to the kinetic profiles, either the recycling and gas-valve sources or the particle confinement time $\tau_{p}$ (used for this work) are required. $\tau_{p}$ allows FRANTIC to calculate the radial outflow of thermal ions, which must be balanced by the ionization of neutrals (fueling). Combined with the neutral penetration probability based on the kinetic profiles, this allows FRANTIC to determine neutral density profiles in absolute units. By varying $\tau_{p}$ in the simulation, a range of radial neutral density profiles are obtained.

\subsection{FIDASIM}

The Monte Carlo code FIDASIM [29] is used to translate fast-ion distribution functions from NUBEAM into synthetic spectra for a given line of sight geometry. The code employs the same kinetic profiles as used by TRANSP, as well as the equilibrium from TRANSP. For TCV, the code was modified to be able to simulate hydrogen beams in combination with a deuterium fast-ion distribution function (the diagnostic beam injects hydrogen neutrals). Furthermore, the simulation of passive FIDA radiation was added due to the previously discussed strong passive FIDA radiation. For the simulation of passive FIDA radiation, the code now uses the background neutral density from FRANTIC and assumes that their velocity is represented by the local ion temperature. By additionally assuming that those neutrals are in the $n=1$ state, charge exchange rates with fast ions can be calculated. The neutralized fast ions are then followed through the 3D simulation grid of FIDASIM and the D-alpha radiation, observed along a given line of sight, can be determined.

Figure 6] shows simulated spectra from FIDASIM for a central toroidal line of sight and a central poloidal line of sight. A significantly stronger passive FIDA signal (blue) is predicted for the toroidal view, compared with the poloidal one. This is expected because the toroidal lines of sight are more tangential to the edge region and have longer paths within the plasma. In contrast, the simulated active FIDA signal (red) is significantly 

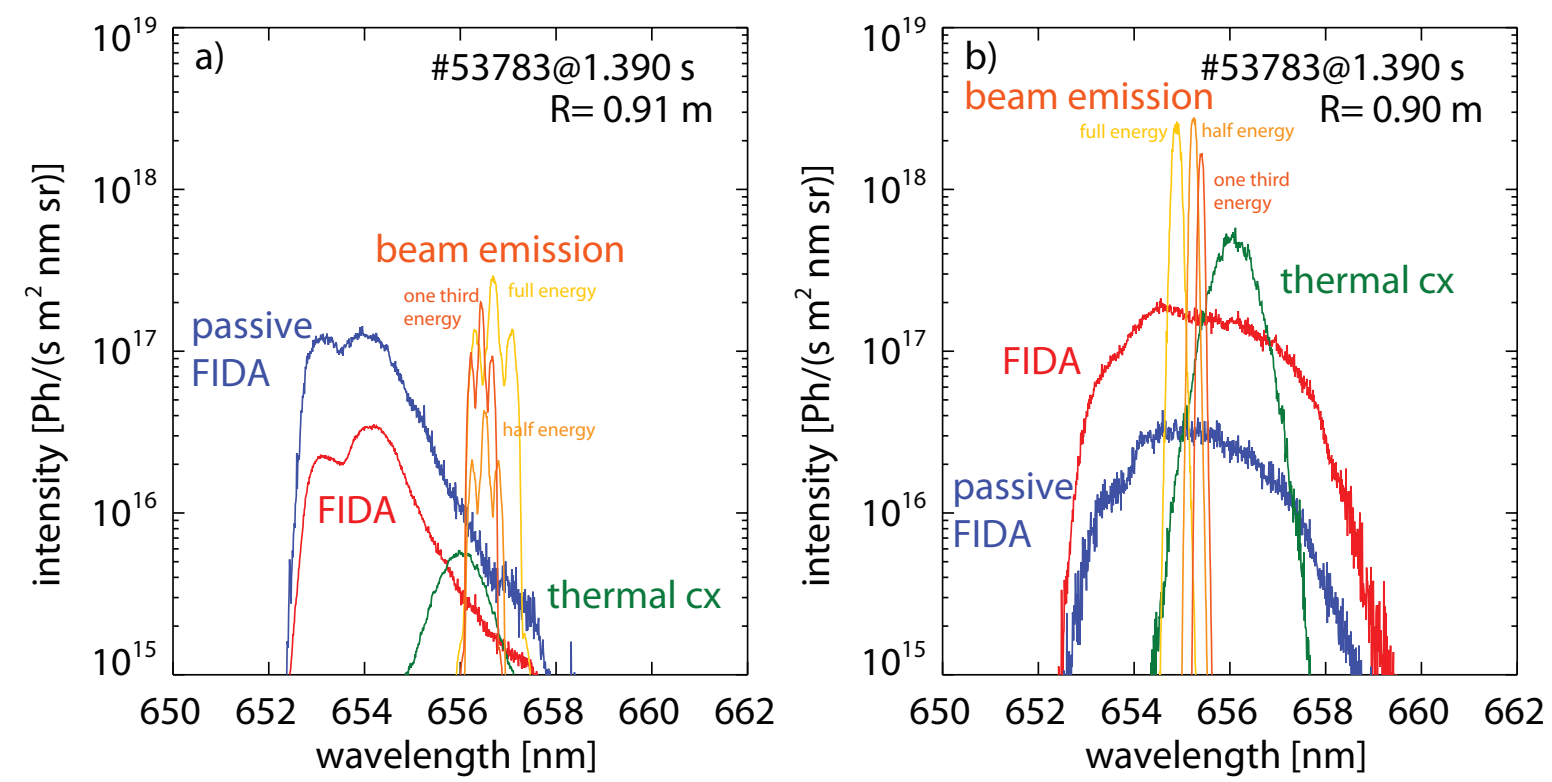

FIG. 6: Synthetic spectra of the toroidal view using the DNB (a) and of the poloidal view using the heating beam (b). The simulated spectra correspond to a TRANSP simulation for which $\tau_{p}=4.5 \mathrm{~ms}$ was assumed.

larger of in the poloidal measurement since the NBI source is considerably stronger than the diagnostic beam. In addition to the FIDA emission, the predicted beam emission, consisting of the full, half and one third energy components is plotted. It exhibits stronger Doppler shifts in the poloidal measurement since the LOS geometry is more tangential to the neutral beam. The thermal charge exchange component is shown in green. Its width corresponds to the ion temperature and it is slightly blue-shifted due to the toroidal plasma rotation.

\section{Analysis of experiments with on- and off-axis NBI}

\subsection{Discharge overview}

Several experiments with $1 \mathrm{MW}$ of NBI heating power were performed with on-axis NBI or with a vertically shifted plasma $(+10 \mathrm{~cm})$ to study off-axis NBI. The discharges were in limiter configuration with a clockwise directed magnetic field of $1.43 \mathrm{~T}$ and a clockwise directed current of $180 \mathrm{kA}$ (seen from above). This corresponds to co-injected NBI with relatively small first orbit losses. Figure 7 shows time traces from two representative discharges (\#53778 and \#53783). The off-axis discharge \#53778 ran through, while the on-axis discharge \#53783 disrupted at $1.5 \mathrm{~s}$ due a problem with the vertical control. The discharges were heated with 1.0 MW of centrally deposited ECRH that additionally drove a counter plasma current. This procedure results in flat or reversed q-profiles 

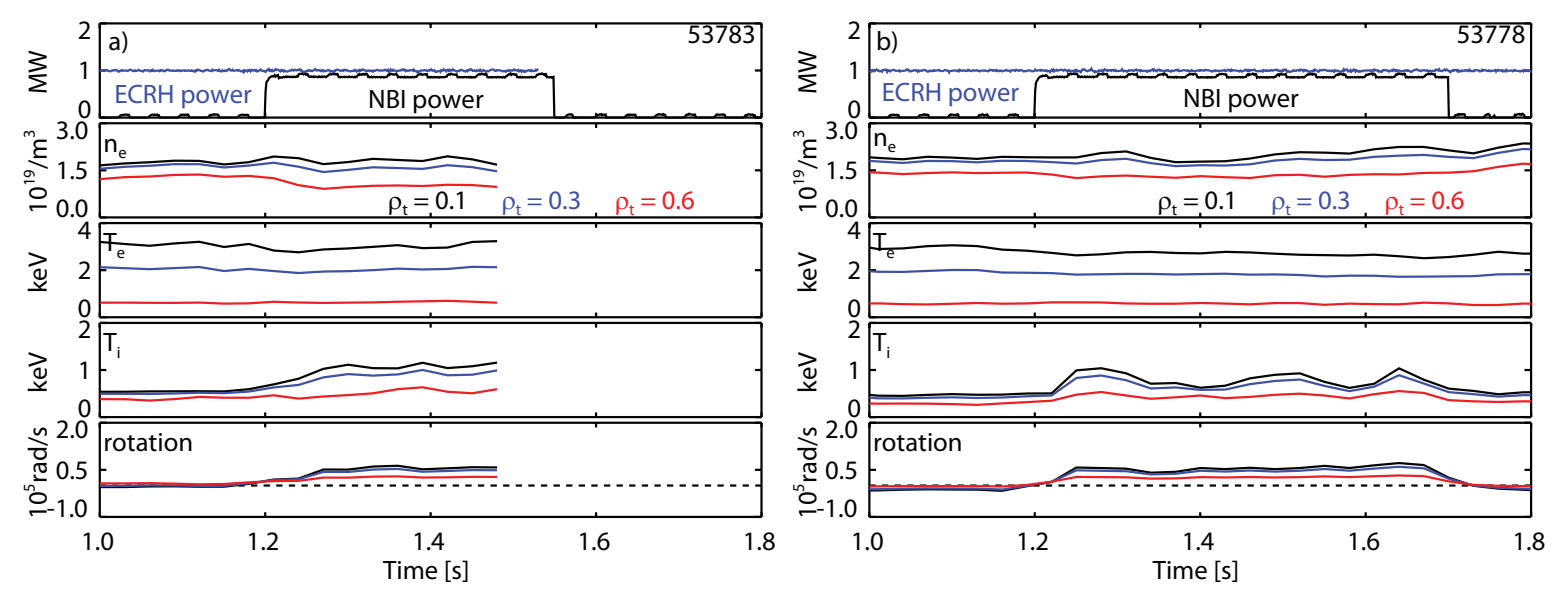

FIG. 7: Representative time traces of discharges \#53783 (on-axis) and \#53778 (off-axis).

and avoids sawtooth instabilities which would adversely affect the fast-ion distribution function. Between $1.2 \mathrm{~s}$ and $1.7 \mathrm{~s}$, the heating beam was injected with $0.9 \mathrm{MW}$ of power (assuming $100 \mathrm{~kW}$ of neutral beam losses in the beam duct). This increases the central ion temperature from $\sim 0.4 \mathrm{keV}$ to $\sim 1 \mathrm{keV}$ and the toroidal plasma rotation frequency from $\sim-10 \mathrm{krad} / \mathrm{s}$ to $\sim 60 \mathrm{krad} / \mathrm{s}$. The electron temperature almost remains unaffected during NBI as ion heating dominates at high electron temperatures. According to [30] the critical energy above which electron heating becomes dominant is 18.6 times the electron temperature. The NBI injection energy $(25 \mathrm{keV})$ is well below this critical energy $(\sim 40$ $\mathrm{keV})$.

In absence of the heating neutral beam, the effective charge profile is typically assumed constant over the radius and in time and can be determined by matching predicted and measured loop voltages during ohmic plasma conditions $\left(Z_{\text {eff }}=3\right)$. During heating beam operation however, a hollow $Z_{\text {eff }}$ profile is expected $\left(Z_{\text {eff }}=3\right.$ at the plasma edge and $Z_{\text {eff }}=1.6$ in the center) which is in qualitative agreement with CXRS measurements (carbon impurity profiles from relative intensities) and also agrees with measurements of the central $Z_{\text {eff }}$ value based on soft X-ray data. This hollowness of $Z_{\text {eff }}$ can be explained since the heating beam fuels the plasma significantly and thus dilutes the impurities.

The global energy confinement time during NBI heating can be estimated by dividing the thermal plasma stored energy $w_{\text {th }}(4.9 \mathrm{~kJ}$ (on-axis) and $4.7 \mathrm{~kJ}$ (off-axis)) by the total absorbed heating power. The latter can be calculated by TRANSP and is $1.1 \mathrm{MW}$ during on-axis NBI and 1.2 MW during off-axis for a well matching simulation (assuming $\tau_{p} \sim 5 \mathrm{~ms}$ as discussed later). This yields an energy confinement time of $4.4 \mathrm{~ms}$ (on-axis) and $4.0 \mathrm{~ms}$ (off-axis), providing a lower boundary for the particle confinement time, $\tau_{p}$, since energy transport is typically higher than particle transport. 

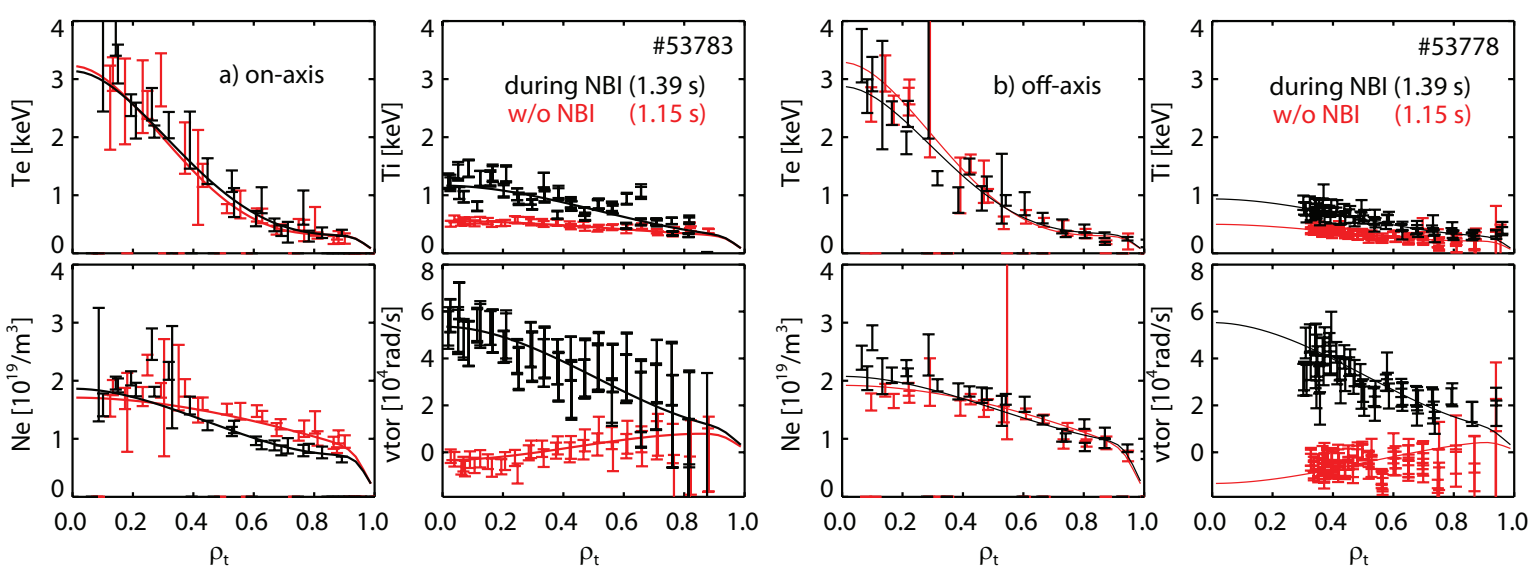

FIG. 8: Kinetic profiles measured in discharges \#53783 and \#53778 with (1.39 s, black) and without NBI (1.15 s, red) which have been fitted for TRANSP with a modified hyperbolic tangent function.

\subsection{TRANSP analysis}

For the interpretation of the discharges, TRANSP simulations were performed assuming either $\tau_{p} \sim 5 \mathrm{~ms}$ or $\tau_{p} \sim 14 \mathrm{~ms}$. $\tau_{p}$ of $\sim 5 \mathrm{~ms}$ is, as mentioned above, slightly above the lower boundary of what can be expected and $\tau_{p} \sim 14 \mathrm{~ms}$ is already a relatively long confinement time. The corresponding background neutral density profiles are displayed in figure 9 in red and green. The predicted neutral densities at the plasma boundary agree with measurements of the absolutely calibrated compact neutral particle analyzer (CNPA), which yields an edge neutral density of $0.8 \times 10^{10} / \mathrm{cm}^{3}$ for the on-axis case and $3 \times 10^{10} / \mathrm{cm}^{3}$ for the off-axis case. The agreement suggests that the predicted neutral densities, an thus the assumed confinement times, $\tau_{p}$, are of the right order of magnitude.

The neutral density profiles in blue in figure 9 correspond to simulations including anomalous fast-ion transport of $0.5 \mathrm{~m}^{2} / \mathrm{s}$ (constant in real and velocity space). The assumption of anomalous fast-ion diffusion increases the neutral density for a given $\tau_{p}$ value because the additional radial fast-ion transport lowers the fast deuterium ion fraction in the plasma. Given that the input total deuterium ion density is fixed, this increases the density of thermal deuterium ions assumed in TRANSP. Combined with the $\tau_{p}$ value, the increased thermal ion density yields an enhanced thermal ion-outflow across the plasma boundary which must be balanced by a larger ionization rate of neutrals. This is why the simulation considering $\tau_{p}=14 \mathrm{~ms}$ exhibits lower neutral densities than the one considering $\tau_{p}=14 \mathrm{~ms}$ plus anomalous fast-ion transport of $0.5 \mathrm{~m}^{2} / \mathrm{s}$.

The assumption of high neutral densities at TCV reduces the fast-ion density due to charge-exchange losses. The fast NBI ions undergo a charge exchange reaction and leave the plasma as neutrals, heating the first wall instead of the plasma. To illustrate the sensitivity of the fast-ion density prediction to $\tau_{p}$, figure 10 shows radial fast-ion density 

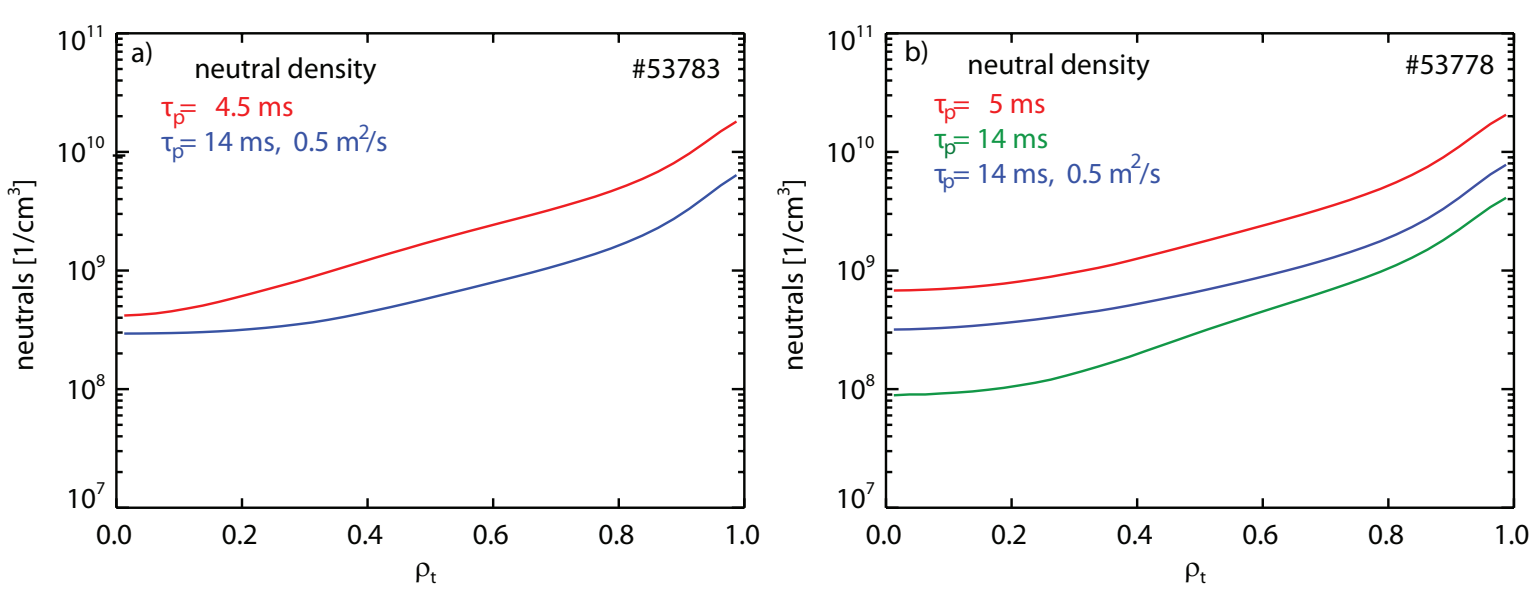

FIG. 9: Predicted radial neutral density profiles for different $\tau_{p}$ values during on-axis NBI (a) and off-axis (b) NBI at $1.39 \mathrm{~s} .0 .5 \mathrm{~m}^{2} / \mathrm{s}$ corresponds to the assumption of additional anomalous fast-ion transport in the TRANSP simulation.
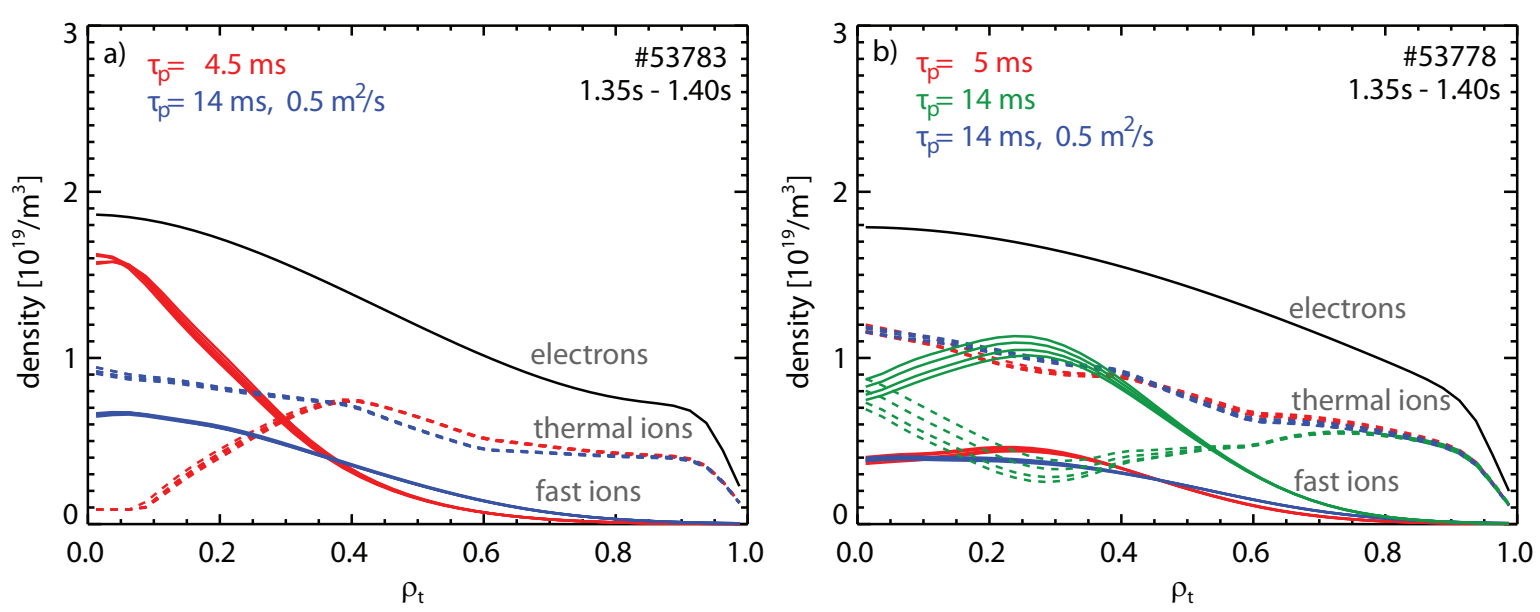

FIG. 10: Electron density profiles (gray) compared with radial fast-ion density profiles assuming $5 \mathrm{~ms}$ of ion-confinement time (red), $14 \mathrm{~ms}$ of ion confinement time (green) and $14 \mathrm{~ms}$ of ion confinement time plus anomalous fast-ion transport. The dashed lines correspond to the calculated thermal Deuterium ion density. 

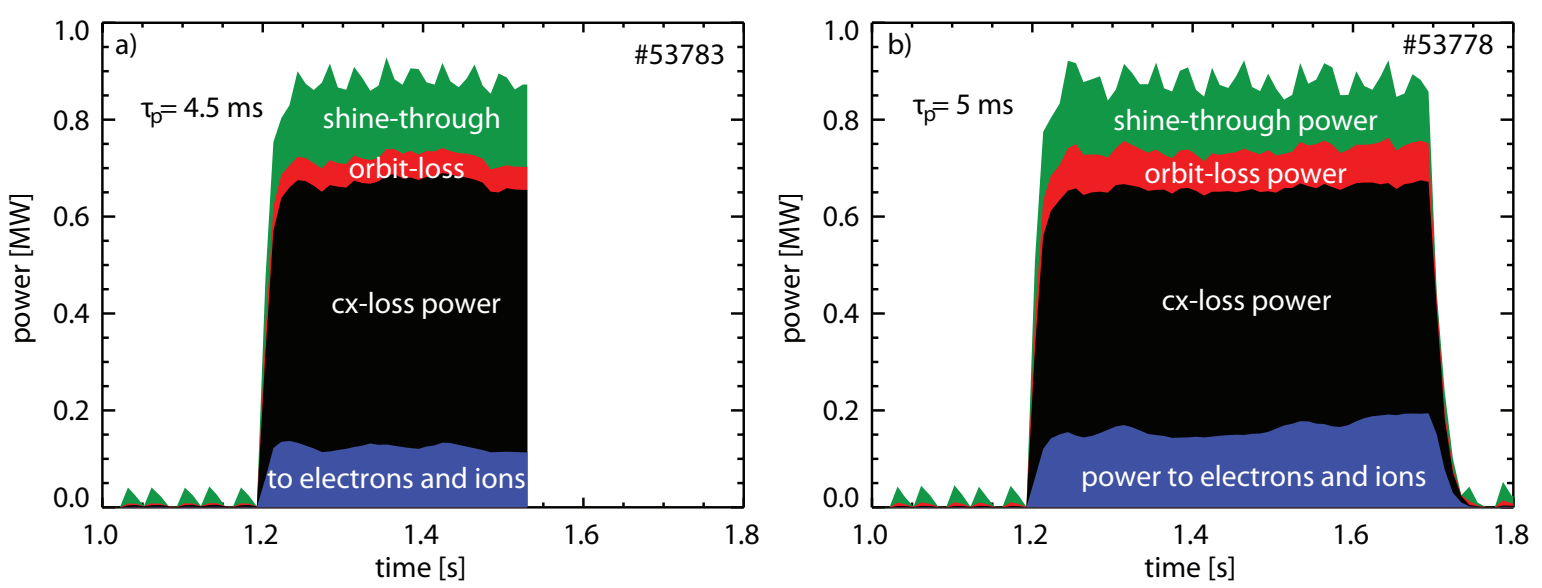

FIG. 11: TRANSP predicted power to electrons and ions, charge exchange losses, orbit losses and shine through power in discharges \#53783 (on-axis) and \#53778 (off-axis).

profiles during on-axis NBI and off-axis NBI assuming $\tau_{p}$ of $\sim 5 \mathrm{~ms}$ and $14 \mathrm{~ms}$. During on-axis NBI, an ever lower value of $\tau_{p}(4.5 \mathrm{~ms})$ was required because $\tau_{p}=5 \mathrm{~ms}$ already caused a stop of the TRANSP simulation as the fast-ion density calculated by NUBEAM exceeded the input total deuterium density (the thermal deuterium ion density becomes negative). For $\tau_{p}=4.5 \mathrm{~ms}$, the central fast-ion density during on-axis NBI reaches more than $80 \%$ of the electron density and, thus, causes the density of thermal deuterium to be close to zero (dashed lines in figure 10).

By choosing additional anomalous fast-ion transport in the simulation $\left(0.5 \mathrm{~m}^{2} / \mathrm{s}\right)$, the fast-ion profiles are flattened significantly, as plotted in blue in figure 10. During off-axis NBI, the simulation exhibits reduced fast-ion densities, compared with the neoclassical simulation $\left(\tau_{p}=14 \mathrm{~ms}\right)$. During on-axis NBI, the simulation with $\tau_{p}=14 \mathrm{~ms}$ becomes possible. The anomalous fast-ion transport reduces the gradient of the fast-ion profile $\left(\rho_{t}: 0.1-0.5\right)$ such that too high fast-ion densities are avoided. Nevertheless, also the simulations including anomalous fast-ion transport exhibit substantial fast-ion fractions $(>20 \%)$.

The reduced fast-ion densities predicted by TRANSP through either strong charge exchange losses or through a combination of charge exchange losses and anomalous fastion transport yield a low heating power of the thermal background plasma. When, e.g. considering $\tau_{p}$ of about $5 \mathrm{~ms}$, up to $500 \mathrm{~kW}$ are lost by charge exchange as indicated in figure 11 (for $\tau_{p}=14 \mathrm{~ms}$, about $350 \mathrm{~kW}$ are lost during off-axis NBI). In addition, orbit losses (about $50 \mathrm{~kW}$ ) and shine-through losses (about $200 \mathrm{~kW}$ ) are considered by TRANSP, which thenyields only about $150 \mathrm{~kW}$ of heating power applied on thermal ions and electrons. This also explains the low total heating power $(\mathrm{NBI}+\mathrm{ECRH})$, applied for the estimation of the energy confinement time, before (1.1 MW during on-axis NBI and 1.2 MW during off-axis). 

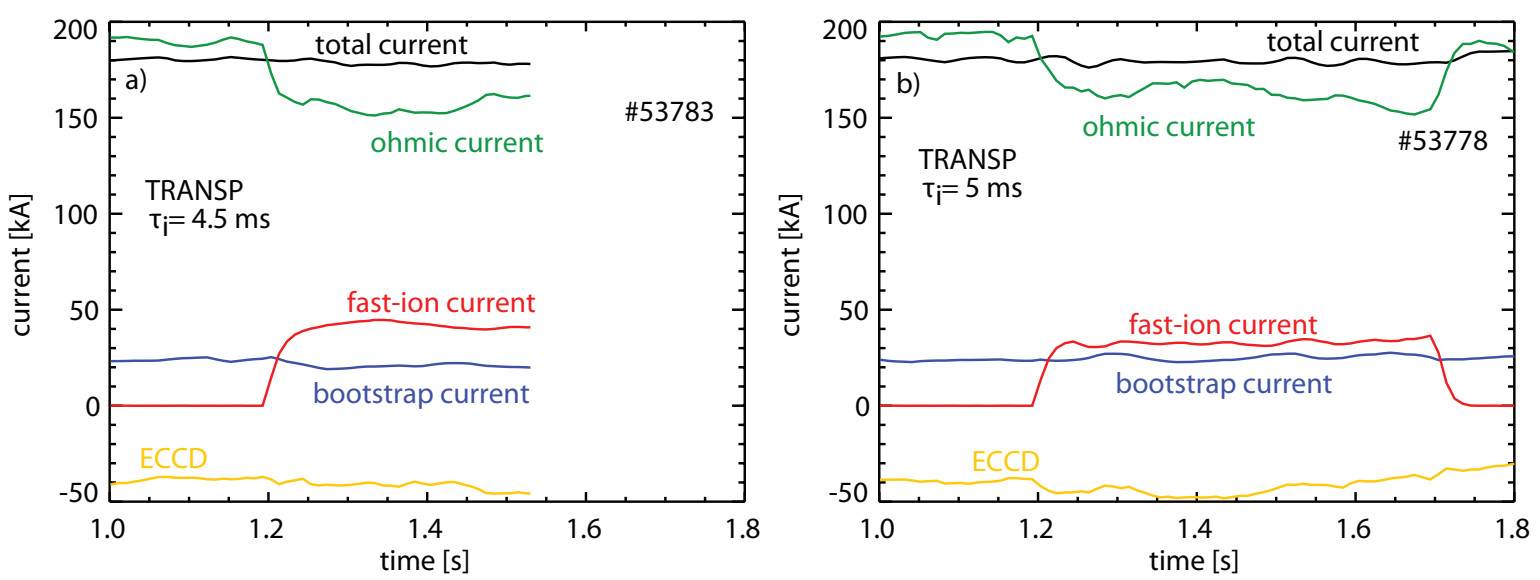

FIG. 12: TRANSP predicted temporal evolution of the different contributions to the plasma current in discharges \#53783 (on-axis) and \#53778 (off-axis).

The low heating power agrees with the modest increase of the measured ion temperature during NBI heating when comparing it with expected ion temperatures. Expected ion temperatures can be determined by assuming that the energy confinement scales like the Kaye l-mode scaling [31] ( $\left.w_{\text {th }} \propto P^{0.28}\right)$ and by assuming that this global scaling holds for the ion channel individually. When considering the low ion-heating predicted by TRANSP with $\tau_{p} \sim 5 \mathrm{~ms}$, the expected ion temperatures are consistent with the measurements. In fact, the expected ion temperatures are even 30\% - 50\% higher than measured. When, instead reducing the fast-ion losses in the simulation by assuming $\tau_{p} \sim 14$ ms during offaxis NBI, the ion heating is significantly higher, yielding up to four times higher expected temperatures (the reduction of the thermal deuterium ion density due to the increased fast-ion population enhances the effect on the exptected ion temperature). The agreement between $\tau_{p} \sim 5 \mathrm{~ms}$ and the increased ion temperature shows that strong fast-ion losses by anomalous transport and/or by charge exchange losses are realistic.

The TRANSP simulation validity can also be assessed by investigating the NBI current drive efficiency and the plasma pressure (toroidal beta). In figure 12, the predicted evolution of the various contributions to the plasma current are displayed for neoclassical simulations assuming $\tau_{p} \sim 5 \mathrm{~ms}$. As can be seen, the bootstrap current and ECCD remain constant throughout the displayed time window while the fast-ion current increases to about $40 \mathrm{kA}$ with NBI heating. During the NBI phase the ohmic current, necessary to maintain the regulated total plasma current, reduces accordingly.

The predicted reduction of the ohmic current is monitored experimentally by the loop voltage, plotted in figure [13. The reduction of the measured loop voltage during NBI is in good agreement with TRANSP for $\tau_{p} \sim 5 \mathrm{~ms}$ and for the simulations that include anomalous fast-ion transport of $0.5 \mathrm{~m}^{2} / \mathrm{s}$ and $\tau_{p} \sim 14 \mathrm{~ms}$. By setting the simulated fastion current to zero, the predicted loop voltages (shown in yellow) do not drop as strongly during the NBI phase. This shows that the drop cannot be solely ascribed to changes 

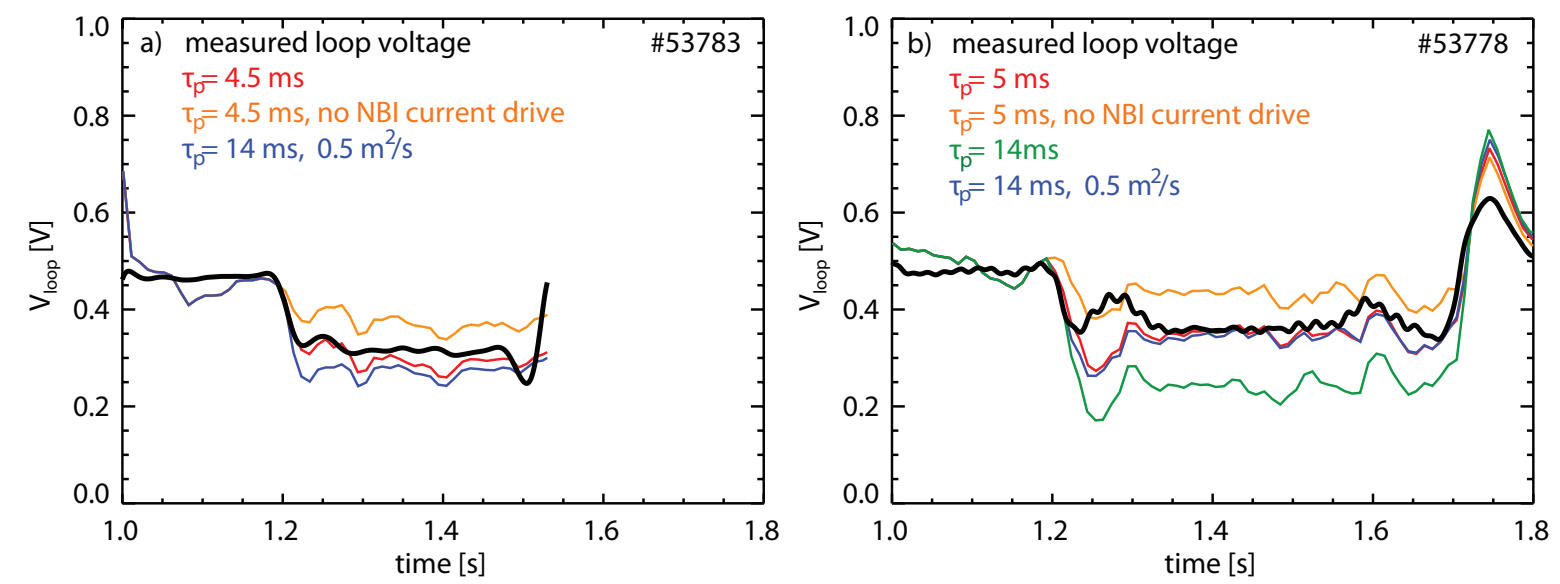

FIG. 13: Measured and predicted loop voltage in discharges \#53783 (on-axis) and discharge \#53778 (off-axis).
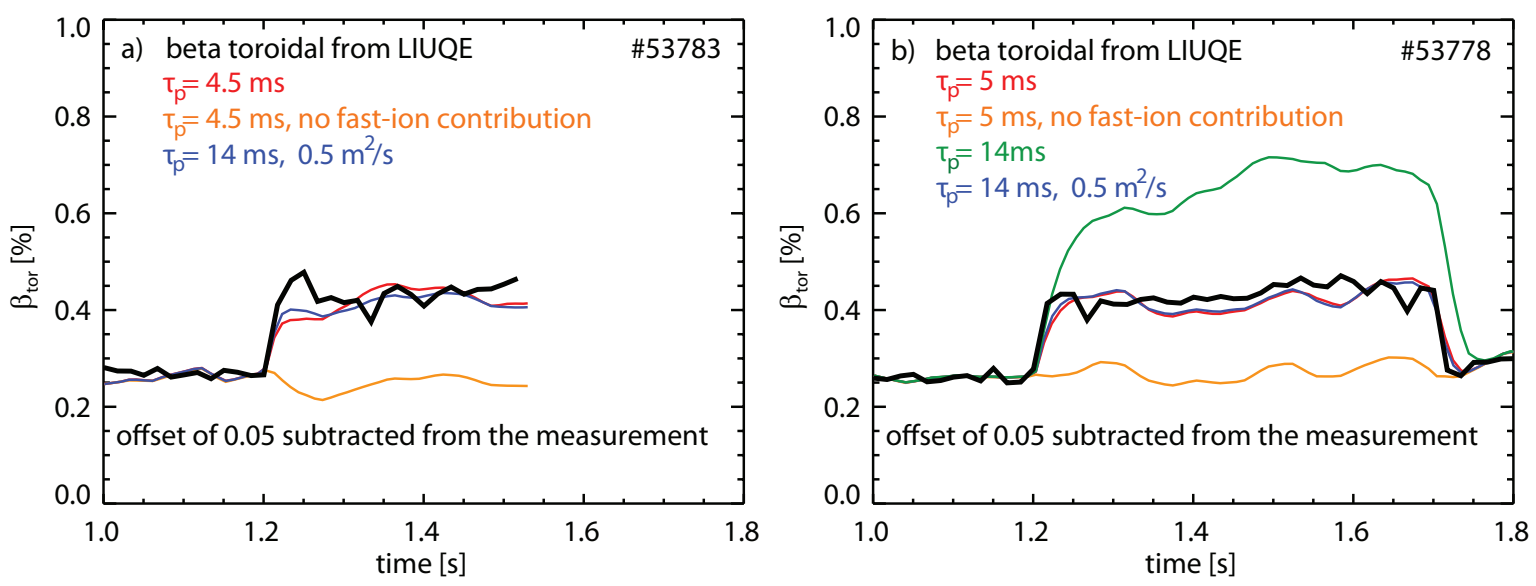

FIG. 14: Measured and predicted toroidal beta in discharges \#53783 (on-axis) and \#53778 (off-axis).

in kinetic profiles (mainly $Z_{\text {eff }}$ ) and, thus, supports an effective fast-ion current drive at TCV.

A similar behavior can be seen in the measured toroidal beta from the equilibrium reconstruction of TCV (called LIUQE [32]), i.e. the ratio of the perpendicular contribution of the plasma pressure to the toroidal magnetic field pressure. Figure 14plots the toroidal beta when switching on NBI. The increase is caused by the fast ions and is well described by the TRANSP simulation for $\tau_{p} \sim 5 \mathrm{~ms}$. No increase is predicted without the fastion contribution (yellow simulations). This implies that the NBI-induced increase of the thermal plasma pressure is marginal. Only the presence of the fast ions increases the toroidal beta significantly. The simulation for $\tau_{p}=14 \mathrm{~ms}$ in combination with anomalous fast-ion transport can also explain the experimental data. However, with $\tau_{p}=14 \mathrm{~ms}$ alone, the loop voltage and the toroidal beta value are overpredicted during off-axis NBI. 


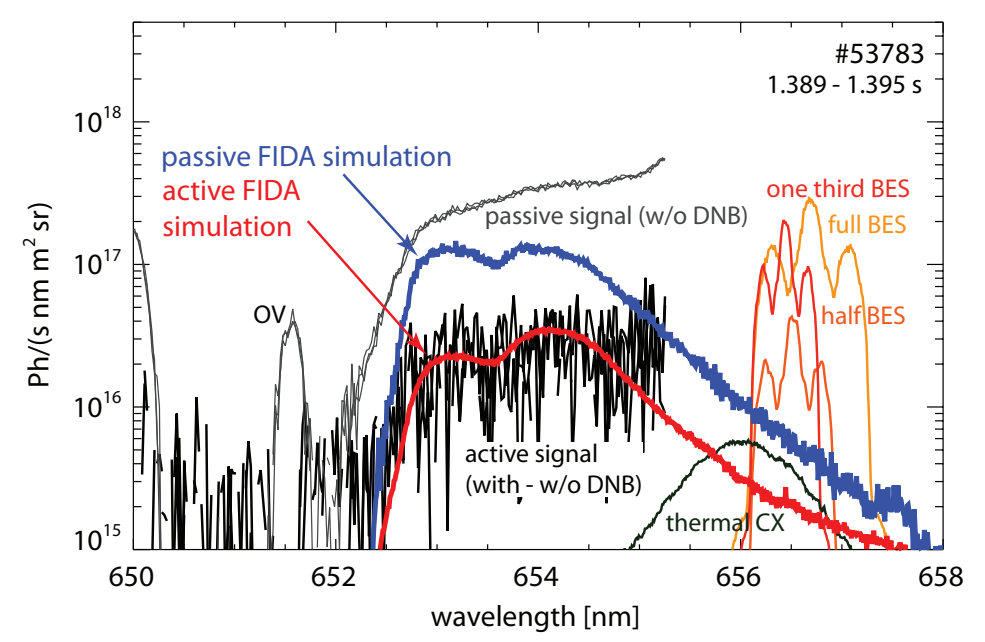

FIG. 15: Active (black) and passive (gray) toroidal FIDA spectrum compared with predictions from FIDASIM.

\subsection{FIDA analysis}

The presence of strong neutral densities that cause charge exchange losses is also supported by the measurement of passive FIDA radiation from the toroidal view. Figure 15 compares an active (black) spectrum with the prediction from FIDASIM that represents the TRANSP simulation with $\tau_{p}=4.5 \mathrm{~ms}$ for discharge \#53783. The active emission corresponds to spectra during DNB operation after subtraction of DNB-free time frames. The toroidal measurement lacks an intensity calibration so the data is scaled (factor $1 \times 10^{13}$ ) to match the active FIDA prediction (red). In addition to the active component, the much stronger passive radiation is plotted in gray. This large passive signal can be reproduced within the right order of magnitude by FIDASIM using the background neutral density from FRANTIC (shown in blue). This demonstrates that the ratio of active to passive radiation is consistently modeled and that large neutral densities exist in these TCV experiments. A factor of two would provide a better match between the measured and synthetic passive signals but remains within the uncertainties of the model: FIDASIM does not consider fast ions outside the plasma boundary neglecting a possible contribution of passive FIDA radiation from that region. Moreover, the neutral density considered by TRANSP and FIDASIM is constant on flux surfaces which is typically not the case in high temperature plasmas. In more advanced simulations, one could, for example, consider an asymmetric neutral density distribution which is significantly higher on the low-field-side than on the high-field-side. Hereby, the total amount of charge exchange losses would not change but the lines of sight of the toroidal view (located at the low-field-side) would collect more passive radiation.

More direct information on the fast-ion density can be obtain for the active measurement using the poloidal view. Figure [16] shows FIDA spectra of two lines of sight 

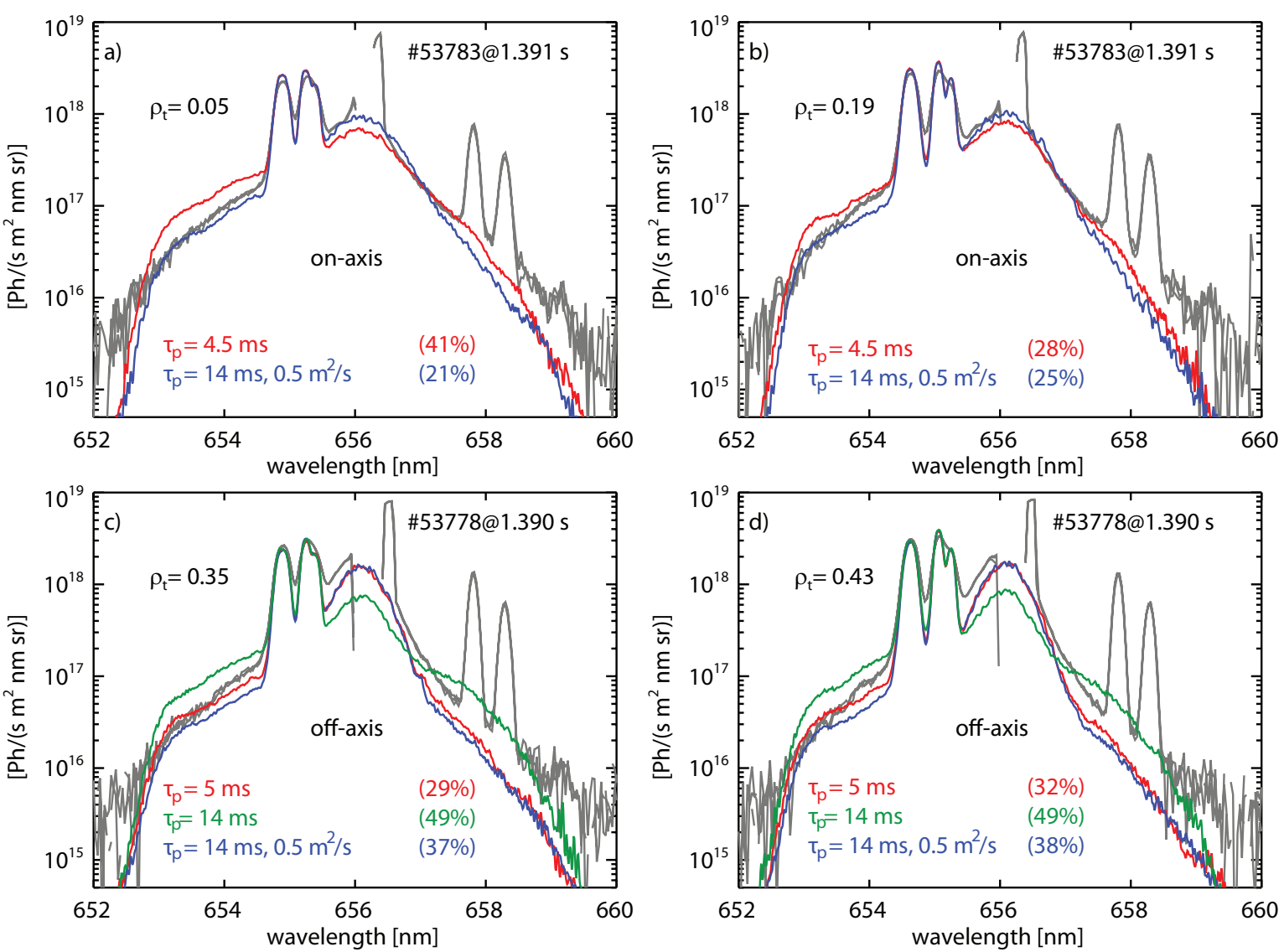

FIG. 16: a) Active FIDA spectra from two poloidal lines of sight during on-axis NBI $(a+b)$ and during off-axis NBI $(c+d)$. In addition, predictions from FIDASIM are plotted that are based on different TRANSP simulations. 
from which the background radiation has been subtracted when the NBI was off. The beam emission, the thermal charge exchange emission and the FIDA emission are clearly present. Moreover, two carbon lines at about $658 \mathrm{~nm}$ are visible despite using background subtraction because these very strong lines did increase during NBI. In addition to the measurement, results from FIDASIM are shown that are based on the TRANSP predicted fast-ion distribution functions. It should be noted that an absolute intensity calibration of the FIDA system is not available due access limitations into the TCV vessel. However, the poloidal measurement could be roughly calibrated by matching the predicted beam emission with the data. One single adjustment factor $\left(1.05 \times 10^{13}\right)$ is used for the whole system which provides good agreement between all channels and the simulated beam emission.

The synthetic spectra plotted in red correspond to the neoclassical simulation assuming $\tau_{p} \sim 5 \mathrm{~ms}$. Apart from the two CII lines at about $658 \mathrm{~nm}$ which are not simulated, this assumption agrees relatively well with the overall shape of the FIDA emission. The average relative difference between the measured intensity, $\mathrm{I}_{\text {meas }}$, and the simulated one, $I_{\text {sim }}$ is displayed in brackets. The difference $\left(\left|I_{\text {meas }}-I_{\text {sim }}\right| / I_{\text {meas }}\right)$ has been calculated by averaging over all pixels while neglecting wavelength regions below $653 \mathrm{~nm}$, above $659 \mathrm{~nm}$, between $655.8 \mathrm{~nm}$ and 656.7 (unshifted D-alpha) and between $657.6 \mathrm{~nm}$ and $658.5 \mathrm{~nm}$ (CII). As can be seen, the average difference during on-axis NBI is about $41 \%$ at $\rho_{t}=0.05$ and $28 \%$ at $\rho_{t}=0.19$. The synthetic FIDA spectra in blue correspond to the TRANSP simulation including anomalous fast-ion transport and yield a relative difference of $21 \%$ and $25 \%$ during on-axis NBI at the two position. This shows that, in particular in the very center $\left(\rho_{t}=0.05\right)$, the assumption of additional anomalous transport yields a better agreement with the measurement, suggesting that anomalous fast-ion transport flattens the radial fast-ion profile during on-axis NBI. During off-axis NBI in contrast, both, the neoclasssical simulation (red) and the simulation assuming anomalous fast-ion transport agree with an average difference of about $30-40 \%$.

In contrast, the neoclassical simulation with $\tau_{p}=14 \mathrm{~ms}$ (green) exhibits larger relative differences (49\%). Here, not only the FIDA signal below $654 \mathrm{~nm}$ and above $658 \mathrm{~nm}$ is poorly matched but also the predicted thermal charge exchange emission in the intermediate region between $655 \mathrm{~nm}$ and $657 \mathrm{~nm}$ is lower than actually measured. The high fast-ion density with $\tau_{p}=14 \mathrm{~ms}$ reduces the thermal ion density, responsible for the thermal charge exchange emission, too much. Instead, the simulations in red and blue yield a better agreement in the region between $655 \mathrm{~nm}$ and $657 \mathrm{~nm}$. The good agreement additionally shows that not only the predicted fast-ion density, but also the assumption of hollow $Z_{\text {eff }}$ profiles is consistent. Higher $Z_{\text {eff }}$ values (e.g. $Z_{\text {eff }}=3$ ) would additionally reduce the density of thermal D-ions and, thus, reduce the predicted thermal charge exchange component. Only the combination of low central $Z_{\text {eff }}$ values and relatively low fast-ion densities (either purely caused by charge exchange losses or caused by weaker change exchange losses in combination with anomalous fast-ion transport) permits good 

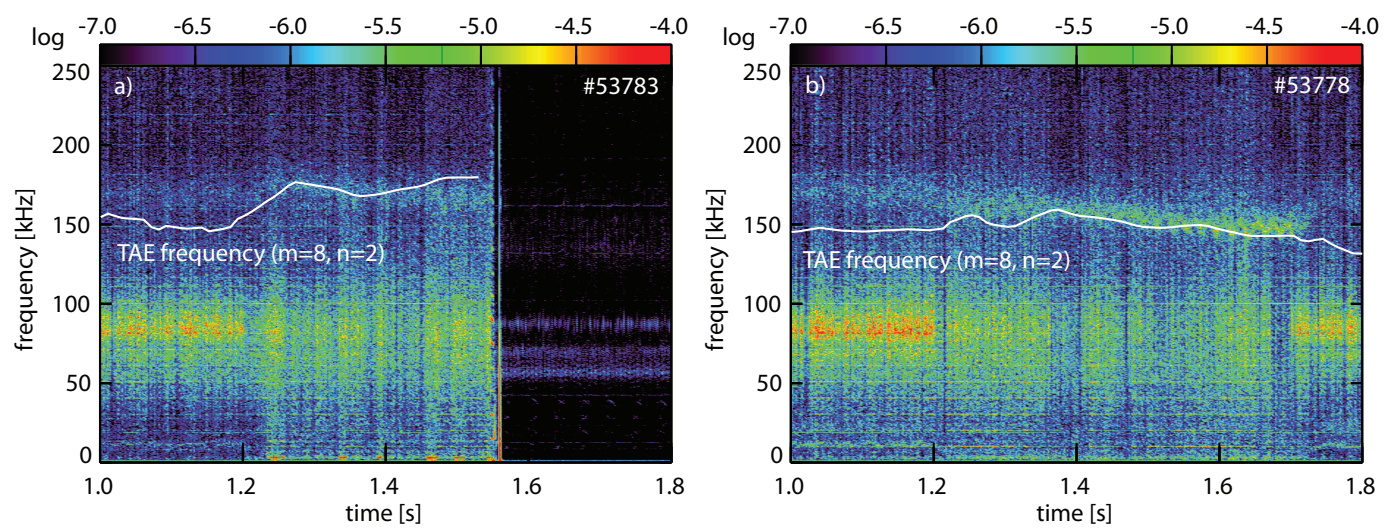

FIG. 17: Magnetic spectrograms for discharges \#53783 (on-axis) and \#53778 (off-axis). The estimated TAE frequency for a mode at the $q=4.25$ surface is plotted in white which would be localized at $\rho_{\mathrm{t}}=0.87$ )

agreement between the measurement and the modelled thermal charge exchange emission.

It should be noted that other combinations of $\tau_{p}$ values and and values for the anomalous diffusion could yield similar or even better agreement with the experimental data. However, the uncertainties of the measurement and the modeling are too large to justify a more detailed scan. In particular modeling the neutral density profile with a 1D code (FRANTIC) instead of 3D, but also the lack of an absolute intensity calibration of the FIDA diagnostic prevents clear conclusions. The presence of anomalous fast-ion transport can, hence, not be confirmed or excluded. However, it is clear based on the FIDA measurement that the fast-ion density is significantly reduced and that charge exchange losses play a major role in those low-density experiments at TCV.

\subsection{Origin of possible anomalous transport}

Concerning the possible anomalous fast-ion transport, high frequency modes might redistribute the fast particles. Figure 17 shows fluctuation spectrograms from magnetic probes [33] for frequencies up to $250 \mathrm{kHz}$. In the frequency band between $50 \mathrm{kHz}$ and $100 \mathrm{kHz}$ a broad-band fluctuation is observed which is most likely related e.g. to control coils of TCV as a clear signal remains in figure 17a after the disruption. At even higher frequencies, a $n=2$ mode is discerned that might be explained by toroidicity induced Alfvén eigenmodes (TAEs). The theoretical frequencies of $(m=8, n=2)$ TAE modes at the $\mathrm{q}=4.25$ surface $\left(\rho_{\mathrm{t}}=0.87\right)$ are plotted in white in figure 17. The theoretical frequencies, 
$\mathrm{f}_{\text {meas }}$ have been calculated including formulas from [34 for non-circular plasmas:

$$
\begin{array}{r}
f_{\text {meas }}=f_{\mathrm{TAE}}+n f_{\text {rot }} \\
f_{\mathrm{TAE}}=\frac{1}{2 \pi} \frac{1}{2 q} \frac{\left|2-\sqrt{1+3 \epsilon^{2} / 4}\right|}{\sqrt{1-\epsilon^{2} / 4}} \frac{v_{a}}{R} 10^{3} \\
\epsilon=\frac{\kappa^{2}-1}{\kappa^{2}+1} \\
v_{a}=\frac{B_{t}}{\sqrt{\mu_{0}\left(n_{D} m_{D}+n_{C} m_{C}\right)}}
\end{array}
$$

Here, $f_{\text {rot }}$ is the plasma rotation frequency in $\mathrm{kHz}, \kappa$ the plasma elongation, $v_{a}$ the Alfvén velocity in $\mathrm{m} / \mathrm{s}, \mathrm{B}_{\mathrm{t}}$ the toroidal field strength in $\mathrm{T}, \mathrm{R}$ the major radius in $\mathrm{m}, n_{D}$ the deuterium ion density in $\mathrm{m}^{-3}, \mathrm{~m}_{\mathrm{D}}$ the deuterium mass in $\mathrm{kg}, \mathrm{n}_{\mathrm{C}}$ the carbon ion density in $\mathrm{m}^{-3}, \mathrm{~m}_{\mathrm{C}}$ the carbon mass in $\mathrm{kg}$ and $\mu_{0}$ the vacuum permeability. As can be seen, the theoretical frequencies for a $(\mathrm{m}=8, \mathrm{n}=2)$ at $\rho_{\mathrm{t}}=0.87$ agree well with the experiment and suggest that NBI heating at TCV drives TAE modes more unstable. However, the modes are weaker but also present before the application of NBI and TAEs are well known to be excited by fast ions with velocities at least in the range of one third of the Alfvén velocity which refers to a fast-ion energy of $77 \mathrm{keV}$ for \#53783 and $64 \mathrm{keV}$ for \#53778. This is well above the NBI injection energy of $25 \mathrm{keV}$, indicating that the modes are more likely driven unstable by fast electrons or turbulence, as observed previously by [35].

In addition to a TAE-induced fast-ion transport, it is well possible that small-scale turbulence affects the fast-ion confinement at TCV. Despite the observation of insignificant turbulence-induced fast-ion transport at other machines [36], the conditions at TCV are different. The high electron temperatures, combined with low fast-ion energies (only $25 \mathrm{keV}$ ) should yield an increased level of fast-ion transport by turbulence [12]. Further studies of the fast-ion transport at TCV, in particular under MHD-quiescent conditions, will therefore be the subject of future investigations.

\section{$5 \quad$ Summary and discussion}

Experiments with $1 \mathrm{MW}$ of nominal NBI power (25 keV, deuterium) have been performed for L-mode plasma configurations with very low electron densities and high electron temperatures. The TRANSP code has been applied to simulate the fast-ion slowing down distribution and to compare predicted loop voltages and toroidal beta values with the measurements. The best agreement with the experiment is obtained by a model including strong fast-ion losses such that only $15 \%$ of the nominal beam power provides heating of the bulk (thermal) plasma. The fast-ion losses are required even to perform TRANSP simulations as, otherwise, the fast-ion density becomes larger than the total deuterium ion 


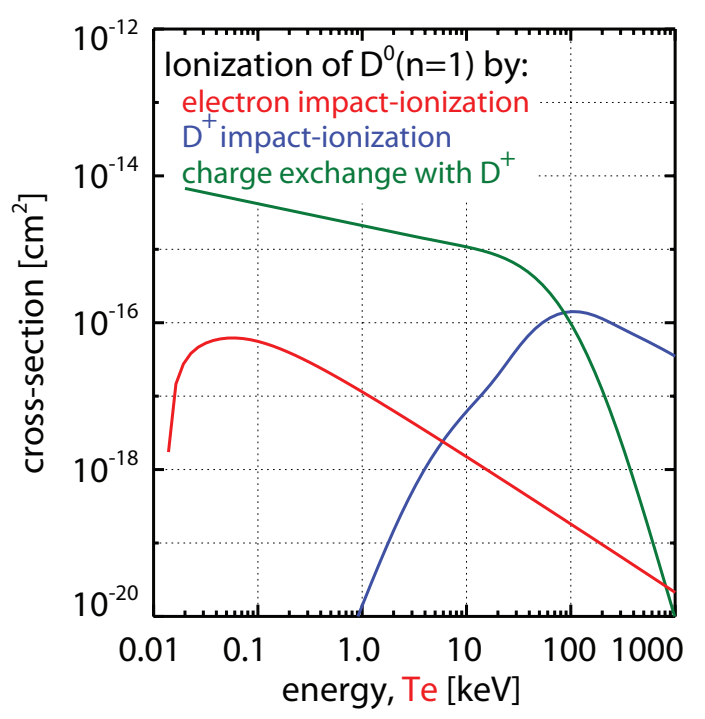

FIG. 18: Cross sections causing the ionization of deuterium neutrals by charge exchange reactions with deuterium ions[38] (green), impact ionization through deuterium ions [39] (blue) and impact ionization through electrons 401 (red). The cross sections for charge exchange and ion impact ionization are given as a function of the relative collision energy. The cross section for electron impact ionization is provided as a function of the electron temperature.

density. The observed weak modification of the ion temperature profiles after switchingon NBI also agrees with the Kaye confinement scaling for L-modes for low effective NBI heating efficiencies. In addition, synthetic spectra from FIDASIM using those TRANSP predictions match active FIDA measurements using a poloidal spectroscopic view.

The required strong fast-ion losses can be modeled in TRANSP by either assuming anomalous fast-ion transport or by considering strong charge exchange losses with background neutrals. The presence of large neutral densities is directly supported by passive FIDA signal levels from toroidal lines of sight and from the absolute neutral fluxes measured by a compact neutral particle analyzer. This shows that charge exchange losses have a significant effect on the fast-ion density and NBI heating efficiency at TCV when the electron density is low. This finding is important, e.g. for future power balance analysis at TCV but also for other devices such as ASDEX Upgrade where charge-exchange losses might appear in the plasma edge that have not yet been considered in the simulations 37.

It should be noted that the fast-ion loss channel by charge exchange reactions competes with the slowing down of fast-ions. When performing experiments with higher densities and lower electron temperatures, the slowing down time of fast-ions can be reduced significantly. Thus, the fast ions might be thermalized before having the chance to undergo charge exchange and the NBI heating efficiency will increase. In addition, higher den- 
sities will attenuate neutrals from the plasma edge more efficiently and therefore reduce the charge exchange losses.

However, fast-ion studies typically aim at maximizing the fast-ion pressure which requires long slowing down times. Therefore, another approach to reduce the neutral density could be to increase the scrape off-layer width and thereby enhance electron impact ionization (ion-impact ionization is negligible at the temperatures reached at TCV and as charge exchange with deuterium ions does not contribute to the reduction of neutrals). As can be seen in figure 18, the cross-section for electron impact ionization of deuterium neutrals $(\mathrm{n}=1)$ peaks at about $50 \mathrm{eV}$ and then strongly decreases towards higher temperatures. Wide scrape of layers with temperatures in the range of $50 \mathrm{eV}$ could, hence, avoid the penetration of neutrals. For future experiments, a diverted plasma configuration with wider scrape of layers could be used (instead of a limiter configuration), which might reduce the penetration of neutrals by enhanced electron impact ionization.

Nevertheless, it is unlikely that charge exchange losses can be reduced to an insignificant amount in TCV. The density of neutrals must therefore be characterized accurately in future investigations to draw clear conclusions on the level of anomalous fast-ion transport. This requires simulations using the EMC3-Eirene [41] code, providing 3D distributions of neutrals, as well as detailed measurements with the neutral particle analyzer at TCV [20]. As soon as the density of neutrals is known accurately enough, ASCOT simulations can be performed with the neutral density as input (ASCOT is currently updated to be able to model charge exchange losses). This will allow one to predict accurate neoclassical fast-ion distribution functions that can be used as input e.g. for the Hagis code. The latter models the interaction between MHD modes and fast particles and thus allows detailed investigations of the fast-ion transport induced by high frequency MHD modes, as observed during NBI heating and tentatively attributed to toroidicity induced Alfvén eigenmodes. Moreover, the impact of turbulence on the fast ions can be addressed by considering fast-ion diffusion coefficients in TRANSP and ASCOT that have been derived e.g. from GENE simulations.

For future experiments, it is even planned to add a toroidal FIDA view that intersects the heating beam and that will be dominated by strong active signals. Due to the better signal to noise ratio, the inference of a $2 \mathrm{D}$ fast-ion velocity distribution function at TCV by velocity-space tomography [42] might become possible. Figure 19] shows a comparison between the TRANSP predicted fast-ion velocity space for discharge \#53783 and a tomographic inversion from two synthetic FIDA spectra. 10\% of Gaussian noise was added to the synthetic spectra and wavelength ranges were discarded where the FIDA light is not observable due to the much brighter thermal charge exchange and beam emission components. For two-view FIDA systems such as the proposed TCV one, the use of prior information is essential. We use the known position of the beam injection peaks at full-, half and one-third energy, the non-negativity of the distribution function, and the absence of FIDA light to restrict the velocity space [43. The overall shape of the distribution func- 

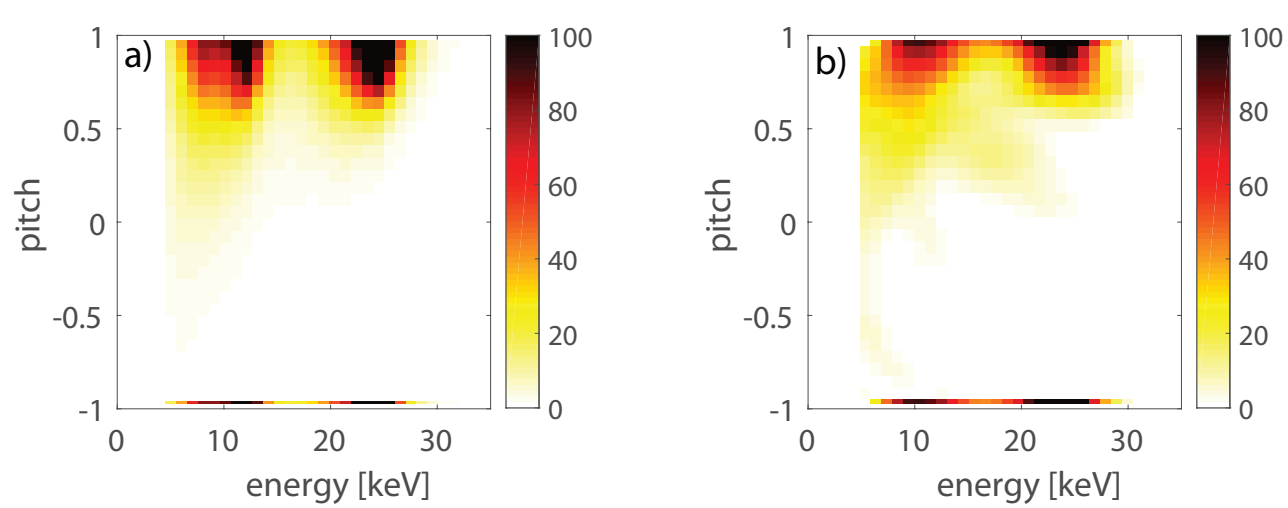

FIG. 19: (a) TRANSP predicted central fast-ion velocity space distribution and (b) tomographic inversion from synthetic measurements of two active FIDA views (poloidal + artificial toroidal view) in the experimentally accessible wavelength ranges with $10 \%$ Gaussian noise.

tion in figure $19 \mathrm{~b}$ is well recovered from the noisy synthetic FIDA measurements in the observable wavelength regions. This indicates that a second toroidal FIDA view on the heating beam will permit to use velocity-space tomography on TCV for future studies.

\section{Acknowledgment}

This work has been carried out within the framework of the EUROfusion Consortium and has received funding from the Euratom research and training programme 2014-2018 under grant agreement No 633053. The views and opinions expressed herein do not necessarily reflect those of the European Commission.

\section{References}

[1] HAWRYLUK, R. et al., Physics of Plasmas Close to Thermonuclear Conditions 1 (1980) 19.

[2] PANKIN, A. et al., Computer Physics Communications 159 (2004) 157.

[3] HEIDBRINK, W. W., Physics of Plasmas 15 (2008) 055501.

[4] GEIGER, B. et al., Nuclear Fusion 54 (2014) 022005.

[5] JACOBSEN, A. S. et al., Plasma Physics and Controlled Fusion 58 (2016) 042002.

[6] HEIDBRINK, W. W. et al., Phys. Rev. Lett. 103 (2009) 175001. 
[7] TURNYANSKIY, M. et al., Nuclear Fusion 49 (2009) 065002.

[8] GUENTER, S. et al., Nuclear Fusion 47 (2007) 920.

[9] GARCIA-MUNOZ, M. et al., Nuclear Fusion 49 (2009) 085014.

[10] ZEELAND, M. V. et al., Nuclear Fusion 56 (2016) 112007.

[11] COLlinS, C. S. et al., Phys. Rev. Lett. 116 (2016) 095001.

[12] HAUFF, T. et al., Phys. Rev. Lett. 102 (2009) 075004.

[13] PINCHES, S. et al., Computer Physics Communications 111 (1998) 133.

[14] JENKO, F. et al., Physics of Plasmas (1994-present) 7 (2000) 1904.

[15] KURKI-SUONIO, T. et al., Nuclear Fusion 49 (2009) 095001.

[16] GOODMAN, T. et al., Nuclear Fusion 48 (2008) 054011.

[17] KARPUSHOV, A. N. et al., Fusion Engineering and Design 9697 (2015) 493 , Proceedings of the 28th Symposium On Fusion Technology (SOFT-28).

[18] KARPUSHOV, A. N. et al., Fusion Engineering and Design 84 (2009) 993 , Proceeding of the 25th Symposium on Fusion Technology(SOFT-25).

[19] BEHN, R. et al., Review of Scientific Instruments 70 (1999) 768.

[20] KARPUSHOV, A. N. et al., Review of Scientific Instruments 77 (2006) 033504.

[21] HEIDBRINK, W. W. et al., Plasma Physics and Controlled Fusion 46 (2004) 1855.

[22] DUVAL, B. P. et al., Physics of Plasmas 15 (2008) 056113.

[23] SALEWSKI, M. et al., Plasma Physics and Controlled Fusion 56 (2014) 105005.

[24] WESTERHOF, E., Rijnhuizen Report (1989) 89.

[25] LIN-LIU, Y. R. et al., Physics of Plasmas 4 (1997) 4179.

[26] SAUTER, O. et al., Physics of Plasmas 6 (1999) 2834.

[27] CODA, S. et al., Nuclear Fusion 43 (2003) 1361.

[28] TAMOR, S., Journal of Computational Physics 40 (1981) 104.

[29] HEIDBRINK, W. et al., Commun. Comput. Phys. 10 (2011) 716.

[30] STIX, T. H., Plasma Physics 14 (1972) 367. 
[31] KAYE, S. et al., Nuclear Fusion 37 (1997) 1303.

[32] MORET, J.-M. et al., Fusion Engineering and Design 91 (2015) 1.

[33] MORET, J.-M. et al., Review of Scientific Instruments 69 (1998) 2333.

[34] FESENYUK, O. P. et al., Physics of Plasmas 20 (2013) 122503.

[35] MARASCHEK, M. et al., Phys. Rev. Lett. 79 (1997) 4186.

[36] PACE, D. C. et al., Physics of Plasmas 20 (2013) 056108.

[37] GEIGER, B. et al., Plasma Physics and Controlled Fusion 57 (2015) 014018.

[38] SUMMERS, H. P., http://www.adas.ac.uk (2004).

[39] MULLANE, M. O., http://www.adas.ac.uk/notes/adas_c09-01.pdf (2009).

[40] JANEV, R. K. et al., B2EIRENE report (2004).

[41] LUNT, T. et al., Plasma Physics and Controlled Fusion 56 (2014) 035009.

[42] SALEWSKI, M. et al., Nuclear Fusion 54 (2014) 023005.

[43] SALEWSKI, M. et al., Nuclear Fusion 56 (2016) 106024. 